\title{
Increased Mortality Risk among Early Stage Hormone Receptor Positive Breast Cancer Patients Who Did Not Receive Adjuvant or Neoadjuvant Therapy
}

\author{
Sacha Satram-Hoang ${ }^{*}$, Alisha Stein ${ }^{2}$, Patricia Cortazar ${ }^{2}$, Faiyaz Momin ${ }^{1}$, Carolina Reyes ${ }^{2}$ \\ ${ }^{1}$ Q.D. Research, Inc., Granite Bay, CA, USA \\ ${ }^{2}$ Genentech, Inc., South San Francisco, CA, USA \\ Email: *sacha@qdresearch.com
}

How to cite this paper: Satram-Hoang, S., Stein, A., Cortazar, P., Momin, F. and Reyes, C. (2019) Increased Mortality Risk among Early Stage Hormone Receptor Positive Breast Cancer Patients Who Did Not Receive Adjuvant or Neoadjuvant Therapy. Journal of Cancer Therapy, 10, 1-20. https://doi.org/10.4236/jct.2019.101001

Received: December 6, 2018

Accepted: January 1, 2019

Published: January 4, 2019

Copyright $\odot 2019$ by authors and Scientific Research Publishing Inc. This work is licensed under the Creative Commons Attribution International License (CC BY 4.0).

http://creativecommons.org/licenses/by/4.0/

\begin{abstract}
Background: Hormone receptor positive (HR+), human epidermal growth factor receptor 2 negative (HER2-) is the most common biologic subtype of breast cancer. Although adjuvant therapy has demonstrated a survival benefit in clinical trials, its use is poorly understood in the real-world among elderly breast cancer patients since age is a barrier to receiving adjuvant therapy. An examination of treatment patterns and outcomes associated with receipt of adjuvant/neoadjuvant therapy among elderly HR + HER2-breast cancer patients was undertaken. Methods. There were 18,470 HR + HER2-breast cancer patients from the linked SEER-Medicare database. Patients were diagnosed with stage I-III disease between 1/1/2007-12/31/2011, $\geq 66$ years, enrolled in Medicare Parts A, B and D, and underwent breast cancer surgery after diagnosis. Time-varying Cox proportional hazards regression assessed overall survival. Results: There were 13,670 (74\%) patients treated with adjuvant/neoadjuvant therapy and $4800(26 \%)$ untreated. Compared to treated patients, untreated patients were older, had earlier stage, lower grade, smaller tumors, poorer performance, higher comorbidity score, and less use of a 21 -gene recurrence score (RS) assay $(\mathrm{p}<0.0001)$. In the survival model, increasing age, stage, tumor size, tumor grade, comorbidity score and poor performance were significantly associated with higher mortality risks, while use of an RS assay was associated with lower risks. The Cox model showed a $48 \%$ higher risk of death in untreated compared to treated patients. In a subset of 8967 patients with stage I disease, tumor size $<2.0 \mathrm{~cm}$ and grade 1/2; untreated patients had a $22 \%$ higher risk of death compared to treated patients. Conclusions: Older patients with favorable clinical characteristics (earlier stage, smaller tumor, lower grade) are less likely to be treated and have a
\end{abstract}


higher risk of death compared to adjuvant/neoadjuvant treated patients. An unmet need among older breast cancer patients persists.

\section{Keywords}

Hormone Receptor Positive Breast Cancer, Elderly Patients, Adjuvant Therapy, Survival

\section{Introduction}

Breast cancer is the most common invasive cancer in women and the second leading cause of death from cancer among women in the United States [1]. One-percent of breast cancer occurs in men [2] [3]. Hormone receptor positive $(\mathrm{HR}+)$, human epidermal growth factor receptor 2 negative (HER2-) is the most common biologic subtype of breast cancer occurring in post-menopausal women and men [3], and accounts for about $73 \%$ of incident cases [4]. About $50 \%$ of women diagnosed with breast cancer are over the age of 65 [5]. Breast cancer mortality has declined over the past few decades because of advances in awareness, earlier detection, and adjuvant treatments [6] [7].

In general, patients with early-stage breast cancer undergo primary breast cancer surgery with or without radiation therapy. The National Comprehensive Cancer Network (NCCN) guidelines recommend adjuvant endocrine therapy for postmenopausal women with $\mathrm{HR}+$ breast cancer for a five-year period following diagnosis [8]. The decision to add chemotherapy to adjuvant endocrine therapy is individualized based on patient factors such as age, tumor size, tumor grade, lymph node involvement, and the results of prognostic multigene assays like the 21-gene Recurrence Score (RS) assay [9] [10] [11]. Recent updates in the American Joint Committee on Cancer Criteria (AJCC) for breast cancer staging manual ( $8^{\text {th }}$ edition) have incorporated biomarkers and prognostic panel data to guide clinical decision-making.

The benefit of adjuvant therapy is poorly understood among older patients with breast cancer since age is a barrier to receiving adjuvant therapy and older patients are underrepresented in clinical trials [12] [13]. Observational studies have demonstrated that breast cancer mortality increases with age [14] and older age and comorbidities are associated with less aggressive treatment in non-metastatic breast cancer [13] [15]. We aimed to contribute to the existing evidence by examining adjuvant therapy patterns and survival outcomes among HR+HER2-, stage I-III patients with breast cancer using the linked Surveillance, Epidemiology, and End Results (SEER)-Medicare database.

\section{Methods}

\subsection{Data Source}

The SEER-Medicare database has been described previously [16]. The SEER 
program, supported by the National Cancer Institute (NCI), collects data from diverse geographic tumor registries and is representative of about $26 \%$ of the U.S. population. The Medicare program is administered by the Centers for Medicare and Medicaid Services (CMS) and covers $97 \%$ of the U.S. population for patients aged 65 years and older. The SEER participants who were diagnosed with cancer at age 65 years or above are matched to their Medicare files through an agreement between the NCI and the CMS resulting in a 93\% match rate. All Medicare beneficiaries receive Part A coverage (inpatient care, skilled nursing, home healthcare and hospice care) and approximately $95 \%$ of beneficiaries subscribe to Part B (outpatient and physician services) and this is combined with the clinical, demographic and cause of death information in SEER. The database linkage used in this study included cancer cases diagnosed until 2011 with their Medicare claims through 2013. Institutional review board (IRB) approval was waived by the New England IRB because the National Institutes of Health's Office of Human Subjects Research has determined that analyses using SEER-Medicare data are exempt from requiring further IRB review and approval.

\subsection{Study Population}

See Figure 1 for the schematic of the inclusion/exclusion process. Patients with a first primary breast cancer diagnosis were identified using SEER cancer site variables labeled "breast" by International Classification of Diseases for Oncology, $3^{\text {rd }}$ edition (ICD-O-3) codes, C50.0-C50.9. We included patients with AJCC stage I, II, or III invasive breast cancer from SEER. Patients, $\geq 66$ years with a first primary breast cancer diagnosed between January 1, 2001 and December 31, 2011 were then linked to their Medicare claims for the years 2000 through 2013. Patients needed to be enrolled in Medicare Parts A and B for a full 12 months prior to diagnosis date and were excluded if breast cancer was diagnosed at the time of death or autopsy, and if enrolled in a health maintenance organization (HMO) any time within the 12 months prior to diagnosis as HMO claims are not available in the dataset.

In July 2006, Medicare coverage was expanded to include prescription drugs under Medicare Part D. We had to further restrict our sample to patients diagnosed between 2007-2011 and enrolled in Part D for at least 1 month in the year after diagnosis date in order to capture patients eligible for adjuvant endocrine therapy. Patients also underwent breast cancer surgery (lumpectomy and mastectomy) within 6 months after diagnosis. Those who received oral or infused neoadjuvant/adjuvant systemic therapy from diagnosis up to 6 months after breast cancer surgery were part of the "treated" group while those who did not were classified as "untreated". The final analytic cohort included 18,470 patients with HR + HER2-breast cancer.

\subsection{Study Variables}

Patient demographics (age, race/ethnicity, marital status, income, education level 
and geographic region) and tumor characteristics (stage, grade, size, and histology) at the time of breast cancer diagnosis were extracted from the SEER registry file. SEER does not contain a measure of functional status, such as Eastern Cooperative Oncology Group (ECOG), we therefore used a proxy for functional status by Davidoff et al. [17]. This surrogate for functional status utilized Medicare claims to identify the following categories of healthcare services that are associated with poor functional status and difficulty in ambulation: oxygen and related respiratory supplies, wheelchair, walkers and supplies, home health services, and hospitalization, skilled nursing, or long-term care facility stays during the 12 months prior to diagnosis date. The National Cancer Institute (NCI) comorbidity index [18] assessed comorbidity burden using diagnosis and procedure codes in the Medicare claims files to identify the 15 non-cancer comorbidities from the Charlson Comorbidity Index [19]. A weight is assigned to each condition based on its 2-year mortality risk and these weights are summed to obtain an index for each patient. The index accounts for the number and severity of the conditions, with higher scores indicating a greater burden of comorbid disease. The NCI comorbidity index was also constructed using claims in the 12 months prior to diagnosis date.

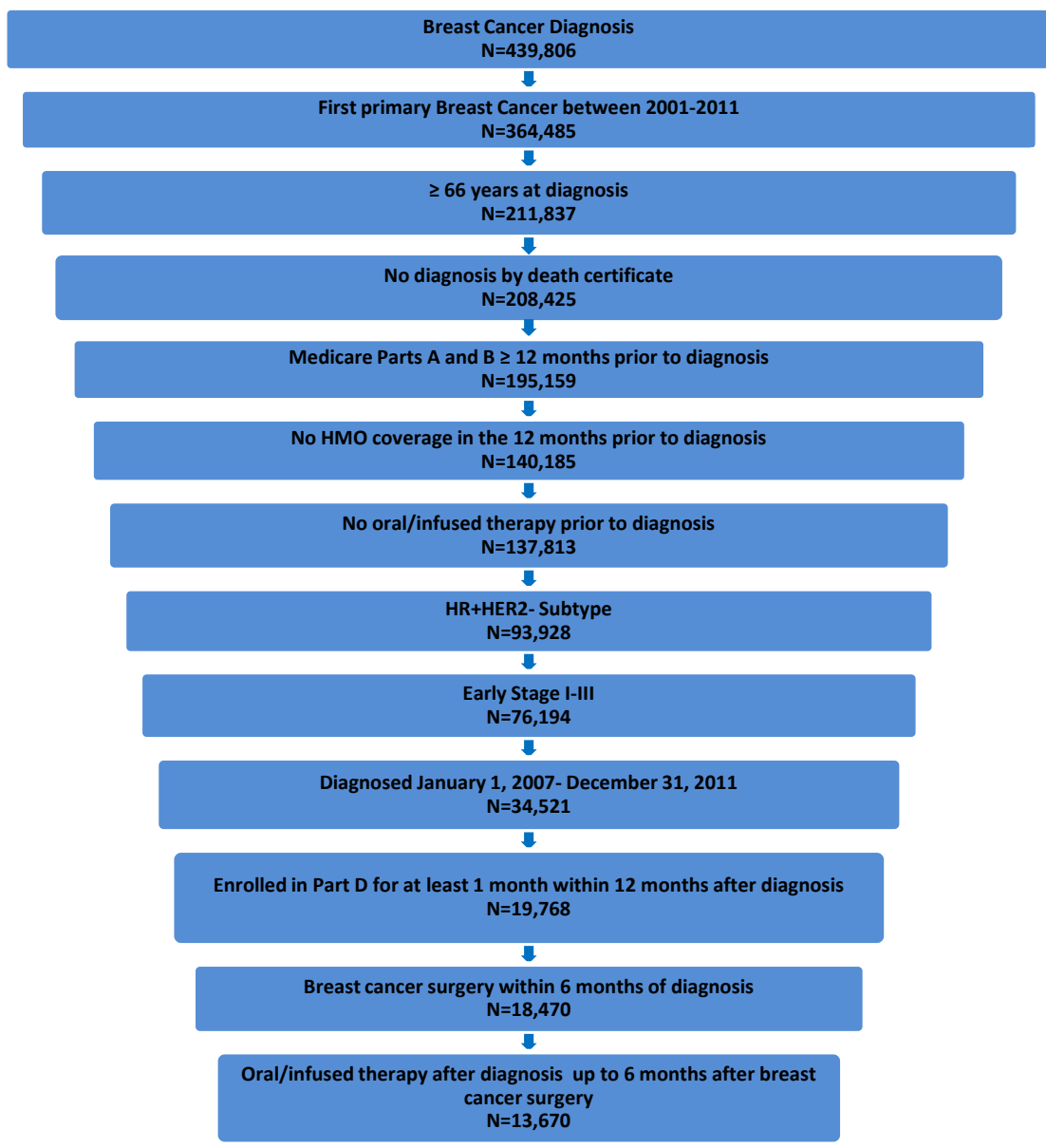

Figure 1. Schematic of inclusion/exclusion process. 
Estrogen receptor (ER) and progesterone receptor (PR) status have been collected by SEER since 1990 and ER and PR results were combined and analyzed jointly as HR status. The year 2010 is the most recent year for which HER2 was available in the dataset. Therefore, we used the presence of Medicare claims for HER2 targeted therapies, i.e., trastuzumab (Herceptin ${ }^{\oplus}$, Roche) and lapatinib (Tykerb, GSK) as a proxy for HER2 positive status and absence of these claims implied HER2 negative status. A case was defined as HR+HER2- if ER and/or PR were positive and HER2 were negative.

Medicare began covering multigene RS assays in February 2006. A modified algorithm from Dinan et al. [20] was used to identify claims for RS assays using Healthcare Common Procedural Coding System (HCPCS) code 84,999 (unlisted chemistry procedure) and provider ID corresponding to Genomic Health within the time period of 2 months before diagnosis to 12 months after diagnosis.

Patients who had breast cancer surgery (lumpectomy and mastectomy) were identified by searching Medicare claims for ICD-9 procedure codes and Current Procedural Terminology (CPT) procedure codes from initial diagnosis date of first primary breast cancer up until 6 months after diagnosis. ICD-9 and CPT procedure codes for radiation therapy within 12 months after diagnosis date were also captured in Medicare claims.

We identified Medicare claims associated with oral hormonal therapy and intravenous chemotherapy from breast cancer diagnosis up to 6 months after breast cancer surgery. The Medicare claims data contain information on each agent administered and date administered, but do not indicate whether the agent is being used in the neoadjuvant, adjuvant, or later-line setting or whether it's part of a sequential or combination regimen. Thus, we developed an algorithm to separate claims into regimen lines. All agents administered preoperatively from diagnosis date were considered to be part of a single neoadjuvant regimen line. Patients were considered to have received adjuvant therapy if administered between breast cancer surgery date and up to 6 months after. Chemotherapy followed by hormonal therapy without a 120-day gap in therapy was considered sequential adjuvant therapy. If a gap in therapy of at least 120 days occurs, then this would indicate the end of adjuvant therapy to avoid capturing treatment for metastatic or recurrent disease.

\subsection{Statistical Analysis}

Baseline characteristics of the study population were described using frequencies and percentages. The Chi-square test for categorical variables and ANOVA or $\mathrm{t}$-test for continuous variables were used to test for differences between groups (treated versus untreated). A $p$-value of $<0.05$ was considered statistically significant. Multivariable logistic regression modeling examined the effect of demographic and clinical factors on the odds of receiving neoadjuvant/adjuvant therapy.

All cause overall survival (OS) was measured from date of breast cancer sur- 
gery to date of death. Death date was captured through 2013 using Medicare enrollment files. If the Medicare date of death was missing, then the SEER date of death was used. Patients were censored at the end of the follow-up period (December 31,2013) or until Medicare claims were no longer available. Crude survival was estimated using Kaplan-Meier survival curves and the log-rank test was applied to compare neoadjuvant/adjuvant treated patients with untreated patients. A time-varying Cox proportional hazards regression model was built to estimate the relative risk of mortality between neoadjuvant/adjuvant treated patients and untreated patients. In the model, neoadjuvant/adjuvant therapy was used as a time-dependent factor to account for variation in treatment initiation between patients and to minimize the introduction of immortal time bias into the analysis (period of follow-up time during which death cannot occur) [21]. To determine the extent to which age plays a role in treatment receipt and prognosis, we conducted a subgroup survival analysis among 8967 patients who had more favorable clinical characteristics (stage I disease, tumor size $<2.0 \mathrm{~cm}$ and tumor grade $1 / 2$ ) to compare neoadjuvant/adjuvant treated with untreated patients.

\section{Results}

\subsection{Treatment Patterns}

There were 13,670 (74\%) patients who received adjuvant/neoadjuvant treatment with hormonal and/or chemotherapy (Treated) and 4800 (26\%) patients who did not (Not Treated). Receipt of adjuvant/neoadjuvant therapy (Treated) increased over the study time-period from $72 \%$ in 2007 to $77 \%$ in 2011 ( $p<0.0001)$. Of the treated patients, $11016(81 \%)$ received hormonal therapy only, 1981 (15\%) received chemotherapy only and lastly, 673 (5\%) received sequential therapy with chemotherapy followed by hormonal therapy. Overall, only $16 \%$ of the study population had a RS assay and the rates of testing increased over the study time-period from $6 \%$ in 2007 to $24 \%$ in 2011 ( $p=0.0002$ ). In regards to treatment rates in association with RS assays; the rates of sequential chemotherapy followed by hormonal therapy decreased from $6 \%$ in 2007 to $5 \%$ in 2011, the use of hormonal therapy increased from $60 \%$ in 2007 to $73 \%$ in 2011 , and chemotherapy only remained consistent at $11 \%$ throughout the time period $(\mathrm{p}=$ 0.0017; data not shown).

\subsection{Patient Characteristics}

Table 1 shows the distribution of patient characteristics by treatment group. The mean age at diagnosis was 75.6 years and approximately $1 \%$ of the cohort were men $(n=170)$. Compared to treated patients, untreated patients were older, with about $60 \%$ over the age of 75 . Untreated patients were also more likely to be widowed $(41.1 \%$ vs. $33.3 \%)$ compared to treated patients. In regards to clinical characteristics, a greater proportion of untreated patients were diagnosed with stage I disease ( $70.3 \%$ vs. $56.2 \%)$, grade 1 tumors (35.0\% vs. $29.1 \%)$ and $71.5 \%$ 
vs. $62.8 \%$ had small tumor size of $<2.0 \mathrm{~cm}$ compared to treated patients. Untreated patients were also less likely to have received radiotherapy, had poorer performance, a higher comorbidity burden, and were less likely to have genomic testing for risk of recurrence $(\mathrm{p}<0.0001)$.

In the adjusted logistic regression model (Table 2), increasing age, being unmarried and having indicators of poor performance decreased the odds of receiving neoadjuvant/adjuvant therapy; while male gender, later stage, higher tumor grade, radiotherapy and genomic testing increased the odds of receiving neoadjuvant/adjuvant therapy.

Table 1. Baseline demographic and clinical characteristics by treatment status.

\begin{tabular}{|c|c|c|c|c|c|c|c|}
\hline \multirow[t]{2}{*}{ Characteristics } & \multicolumn{2}{|c|}{$\begin{array}{c}\text { Total } \\
\mathrm{N}=18,470\end{array}$} & \multicolumn{2}{|c|}{$\begin{array}{c}\text { Treated } \\
\mathrm{N}=13,670\end{array}$} & \multicolumn{2}{|c|}{$\begin{array}{l}\text { Not Treated } \\
\mathrm{N}=4800\end{array}$} & \multirow[t]{2}{*}{$p$-value } \\
\hline & $\mathrm{n}$ & $\%$ & $\mathrm{n}$ & $\%$ & $\mathbf{n}$ & $\%$ & \\
\hline \multicolumn{8}{|l|}{ Age at Diagnosis } \\
\hline $66-70$ & 5423 & 29.4 & 4466 & 32.7 & 957 & 19.9 & $<0.0001$ \\
\hline $71-75$ & 4619 & 25.0 & 3676 & 26.9 & 943 & 19.6 & \\
\hline $76-80$ & 3871 & 21.0 & 2810 & 20.6 & 1061 & 22.1 & \\
\hline$>80$ & 4557 & 24.7 & 2718 & 19.9 & 1839 & 38.3 & \\
\hline Mean Age (95\% CI) & 75.55 & $75.4-75.6$ & 74.67 & $74.6-74.8$ & 78.03 & $77.8-78.2$ & $<0.0001$ \\
\hline \multicolumn{8}{|l|}{ Sex } \\
\hline Male & 170 & 0.9 & 140 & 1.0 & 30 & 0.6 & 0.0127 \\
\hline Female & 18,300 & 99.1 & 13,530 & 99.0 & 4770 & 99.4 & \\
\hline \multicolumn{8}{|l|}{ Race/ethnicity } \\
\hline White & 15,945 & 86.3 & 11,742 & 85.9 & 4203 & 87.6 & 0.0006 \\
\hline Black & 1269 & 6.9 & 941 & 6.9 & 328 & 6.8 & \\
\hline Other/Unknown & 1256 & 6.8 & 987 & 7.2 & 269 & 5.6 & \\
\hline \multicolumn{8}{|l|}{ Marital Status } \\
\hline Single & 1648 & 8.9 & 1204 & 8.8 & 444 & 9.3 & $<0.0001$ \\
\hline Married & 7525 & 40.7 & 5857 & 42.8 & 1668 & 34.8 & \\
\hline Separated/Divorced & 2001 & 10.8 & 1483 & 10.8 & 518 & 10.8 & \\
\hline Widowed & 6525 & 35.3 & 4553 & 33.3 & 1972 & 41.1 & \\
\hline Unknown & 771 & 4.2 & 573 & 4.2 & 198 & 4.1 & \\
\hline \multicolumn{8}{|l|}{$\begin{array}{l}\% \text { of adults with some } \\
\text { college education }\end{array}$} \\
\hline $0-50$ & 5376 & 29.1 & 4123 & 30.2 & 1253 & 26.1 & $<0.0001$ \\
\hline $51-100$ & 12,751 & 69.0 & 9306 & 68.1 & 3445 & 71.8 & \\
\hline Unknown & 343 & 1.9 & 241 & 1.8 & 102 & 2.1 & \\
\hline \multicolumn{8}{|l|}{ Median Income Quartiles } \\
\hline 1-Low & 4532 & 24.5 & 3449 & 25.2 & 1083 & 22.6 & 0.0013 \\
\hline 2 & 4532 & 24.5 & 3294 & 24.1 & 1238 & 25.8 & \\
\hline 3 & 4529 & 24.5 & 3321 & 24.3 & 1208 & 25.2 & \\
\hline 4-High & 4531 & 24.5 & 3364 & 24.6 & 1167 & 24.3 & \\
\hline
\end{tabular}




\section{Continued}

\begin{tabular}{|c|c|c|c|c|c|c|c|}
\hline Unknown & 346 & 1.9 & 242 & 1.8 & 104 & 2.2 & \\
\hline \multicolumn{8}{|c|}{ Geographic region } \\
\hline Midwest & 1467 & 7.9 & 1047 & 7.7 & 420 & 8.8 & \multirow[t]{4}{*}{0.0003} \\
\hline Northeast & 1042 & 5.6 & 815 & 6.0 & 227 & 4.7 & \\
\hline South & 7076 & 38.3 & 5289 & 38.7 & 1787 & 37.2 & \\
\hline West & 8885 & 48.1 & 6519 & 47.7 & 2366 & 49.3 & \\
\hline \multicolumn{8}{|c|}{ Stage at Diagnosis } \\
\hline Stage I & 11,061 & 59.9 & 7687 & 56.2 & 3374 & 70.3 & \multirow[t]{3}{*}{$<0.0001$} \\
\hline Stage II & 5908 & 32.0 & 4699 & 34.4 & 1209 & 25.2 & \\
\hline Stage III & 1501 & 8.1 & 1284 & 9.4 & 217 & 4.5 & \\
\hline \multicolumn{8}{|l|}{ Histology } \\
\hline Ductal & 13,017 & 70.5 & 9673 & 70.8 & 3344 & 69.7 & \multirow[t]{4}{*}{$<0.0001$} \\
\hline Lobular & 2242 & 12.1 & 1737 & 12.7 & 505 & 10.5 & \\
\hline Mixed & 2036 & 11.0 & 1498 & 11.0 & 538 & 11.2 & \\
\hline Others & 1175 & 6.4 & 762 & 5.6 & 413 & 8.6 & \\
\hline \multicolumn{8}{|l|}{ Tumor Grade } \\
\hline Grade 1 & 5659 & 30.6 & 3977 & 29.1 & 1682 & 35.0 & \multirow[t]{4}{*}{$<0.0001$} \\
\hline Grade 2 & 8990 & 48.7 & 6740 & 49.3 & 2250 & 46.9 & \\
\hline Grade $3 / 4$ & 3083 & 16.7 & 2432 & 17.8 & 651 & 13.6 & \\
\hline Unknown & 738 & 4.0 & 521 & 3.8 & 217 & 4.5 & \\
\hline \multicolumn{8}{|l|}{ Tumor Size } \\
\hline$<2.0 \mathrm{~cm}$ & 12,010 & 65.0 & 8580 & 62.8 & 3430 & 71.5 & \multirow[t]{4}{*}{$<0.0001$} \\
\hline $2.0-4.9 \mathrm{~cm}$ & 5499 & 29.8 & 4319 & 31.6 & 1180 & 24.6 & \\
\hline$\geq 5.0 \mathrm{~cm}$ & 919 & 5.0 & 739 & 5.4 & $190^{\mathrm{a}}$ & 4.0 & \\
\hline Unknown & 42 & 0.2 & 32 & 0.2 & 190 & 4.0 & \\
\hline \multicolumn{8}{|c|}{$\begin{array}{l}\text { Poor Performance } \\
\text { Indictors }\end{array}$} \\
\hline No & 16,350 & 88.5 & 12,291 & 89.9 & 4059 & 84.6 & \multirow[t]{2}{*}{$<0.0001$} \\
\hline Yes & 2120 & 11.5 & 1379 & 10.1 & 741 & 15.4 & \\
\hline \multicolumn{8}{|c|}{ NCI Comorbidity Score } \\
\hline 0 & 10,481 & 56.7 & 7876 & 57.6 & 2605 & 54.3 & \multirow[t]{4}{*}{$<0.0001$} \\
\hline 1 & 4661 & 25.2 & 3444 & 25.2 & 1217 & 25.4 & \\
\hline 2 & 1868 & 10.1 & 1352 & 9.9 & 516 & 10.8 & \\
\hline$\geq 3$ & 1460 & 7.9 & 998 & 7.3 & 462 & 9.6 & \\
\hline \multicolumn{8}{|l|}{ RS Assay } \\
\hline No & 15,460 & 83.7 & 11,066 & 81.0 & 4394 & 91.5 & \multirow[t]{2}{*}{$<0.0001$} \\
\hline Yes & 3010 & 16.3 & 2604 & 19.0 & 406 & 8.5 & \\
\hline \multicolumn{8}{|c|}{$\begin{array}{l}\text { Radiation within } \\
1 \text { year of diagnosis }\end{array}$} \\
\hline No & 7785 & 42.1 & 5301 & 38.8 & 2484 & 51.8 & \multirow[t]{2}{*}{$<0.0001$} \\
\hline Yes & 10,685 & 57.9 & 8369 & 61.2 & 2316 & 48.3 & \\
\hline
\end{tabular}

${ }^{a}$ Cells with counts of less than 11 are combined in compliance with the National Cancer Institute data use agreement for small cell sizes. 
Table 2. Logistic regression model of factors associated with the odds of not receiving neoadjuvant/adjuvant treatment.

\begin{tabular}{|c|c|c|c|c|}
\hline \multirow[t]{2}{*}{ Characteristic } & \multicolumn{4}{|c|}{$\begin{array}{l}\text { All Patients }^{\mathrm{a}} \\
\mathrm{N}=18,470\end{array}$} \\
\hline & $\mathbf{N}$ & OR & $95 \% \mathrm{CI}$ & $p$ value \\
\hline \multicolumn{5}{|l|}{ Age at Diagnosis } \\
\hline $66-70$ (ref) & 5423 & & & \\
\hline $71-75$ & 4619 & 1.14 & $1.028-1.262$ & $<0.0001$ \\
\hline $76-80$ & 3871 & 1.58 & $1.426-1.758$ & 0.0232 \\
\hline$>80$ & 4557 & 2.61 & $2.353-2.897$ & $<0.0001$ \\
\hline \multicolumn{5}{|l|}{ Sex } \\
\hline Female (ref) & 18,300 & & & \\
\hline Male & 170 & 0.61 & $0.403-0.922$ & 0.0190 \\
\hline \multicolumn{5}{|l|}{ Race/ethnicity } \\
\hline White (ref) & 15,945 & & & \\
\hline Black & 1269 & 1.08 & $0.936-1.25$ & 0.0021 \\
\hline Others & 1256 & 0.71 & $0.615-0.828$ & $<0.0001$ \\
\hline \multicolumn{5}{|l|}{ Marital Status } \\
\hline Married (ref) & 7525 & & & \\
\hline Separated/Divorced & 2001 & 1.18 & $1.05-1.334$ & 0.0913 \\
\hline Single & 1648 & 1.20 & $1.062-1.374$ & 0.0476 \\
\hline Widowed & 6525 & 1.09 & $1.001-1.186$ & 0.9867 \\
\hline Unknown & 771 & 0.99 & $0.826-1.185$ & 0.1753 \\
\hline \multicolumn{5}{|l|}{ Stage at Diagnosis } \\
\hline Stage I (ref) & 11,061 & & & \\
\hline Stage II & 5908 & 0.51 & $0.450-0.572$ & 0.1233 \\
\hline Stage III & 1501 & 0.31 & $0.253-0.376$ & $<0.0001$ \\
\hline \multicolumn{5}{|l|}{ Grade } \\
\hline Grade 1 (ref) & 5659 & & & \\
\hline Grade 2 & 8990 & 0.91 & $0.841-0.985$ & 0.2243 \\
\hline Grade 3/4 & 3083 & 0.80 & $0.714-0.897$ & 0.0001 \\
\hline Unknown & 738 & 1.10 & $0.919-1.317$ & 0.0241 \\
\hline \multicolumn{5}{|l|}{ Tumor Size } \\
\hline$<2.0 \mathrm{~cm}$ & 12,010 & & & \\
\hline $2.0-4.9 \mathrm{~cm}$ & 5499 & 1.06 & $0.935-1.197$ & 0.0674 \\
\hline$\geq 5.0 \mathrm{~cm}$ & 919 & 1.21 & $0.968-1.503$ & 0.6109 \\
\hline Unknown & 42 & 2.10 & $0.984-4.501$ & 0.0840 \\
\hline \multicolumn{5}{|l|}{ Poor Performance } \\
\hline Indicators & & & & \\
\hline
\end{tabular}




\section{Continued}

\begin{tabular}{ccccc}
\hline $\begin{array}{c}\text { No (ref) } \\
\text { Yes }\end{array}$ & 16,350 & 1.34 & $1.195-1.492$ & $<0.0001$ \\
NCI Comorbidity Score & 2120 & & & \\
0 (ref) & 10,481 & & & \\
1 & 4661 & 0.98 & $0.900-1.065$ & 0.1715 \\
2 & 1868 & 1.00 & $0.885-1.125$ & 0.5390 \\
$\geq 3$ & 1460 & 1.13 & $0.989-1.296$ & 0.0463 \\
RS Assay & & & & \\
No (ref) & 15,460 & & & \\
Yes & 3010 & 0.53 & $0.45-0.66$ & $<0.0001$ \\
Radiotherapy & & & & \\
No (ref) & 7785 & & & \\
Yes & 10,685 & 0.70 & $0.648-0.751$ & $<0.0001$ \\
\hline
\end{tabular}

${ }^{\text {a }}$ Model also includes geographic region, education, income, year of diagnosis, and histology.

In a subgroup analysis comparing treated patients who received hormonal therapy, chemotherapy-only, and sequential therapy (chemotherapy followed by hormonal therapy); patients who received hormonal therapy were more likely to be older than 75 ( $46 \%$ vs. $21 \%$ and $22 \%$; p < 0.0001$)$, more likely widowed $(35 \%$ vs. $26 \%$ and $27 \%$; $\mathrm{p}<0.0001)$, have stage I disease $(64 \%$ vs. $23 \%$ and $26 \%$; $<<$ $0.0001)$, tumor grade I $(32 \%, 16 \%$ and $16 \%$; $<<0.0001)$, tumor size $<2.0 \mathrm{~cm}$ (68\% vs. $40 \%$ and $41 \%$; p $<0.0001$ ), poorer performance ( $11 \%$ vs. $6 \%$ and $7 \%$; p $<0.0001)$, and NCI comorbidity score $\geq 2$ ( $18 \%$ vs. $14 \%$ and $16 \% ; \mathrm{p}=0.0010)$ compared to patients who received chemotherapy-only and sequential therapy respectively (data not shown). Patients who received chemotherapy-only were more likely to have had radiotherapy $(73 \%$ vs. $59 \%$ and $57 \%$; $p<0.0001)$ compared to patients who received hormonal therapy and sequential therapy.

\subsection{Survival Outcomes}

The mean and median follow-up time for the overall cohort was 48.3 months (95\% CI: 48.1 - 48.6) and 47.6 months (95\% CI: 32.9 - 64.1) respectively. The median overall survival time was not reached in the analysis and the mean unadjusted OS from all cause of death was 65.4 months (95\% CI: 65.1 - 65.6) for the overall population. As shown in Figure 2, the mean unadjusted OS was longer for treated patients (66.6 months; $95 \%$ CI: 66.3 - 66.8) compared to untreated patients (61.5 months; 95\% CI: 61.0- 62.1; log rank $\mathrm{p}<0.0001)$. The mean unadjusted OS was similar for patients treated with hormonal therapy (65.0 months; 95\% CI: 64.7 - 65.3) and chemotherapy only (65.3 months; $95 \%$ CI: 64.6 - 66.1) and slightly shorter for sequential therapy (60.3 months; $95 \% \mathrm{CI}$ : $59.1-61.5), \log$ rank $\mathrm{p}=0.3274$. 


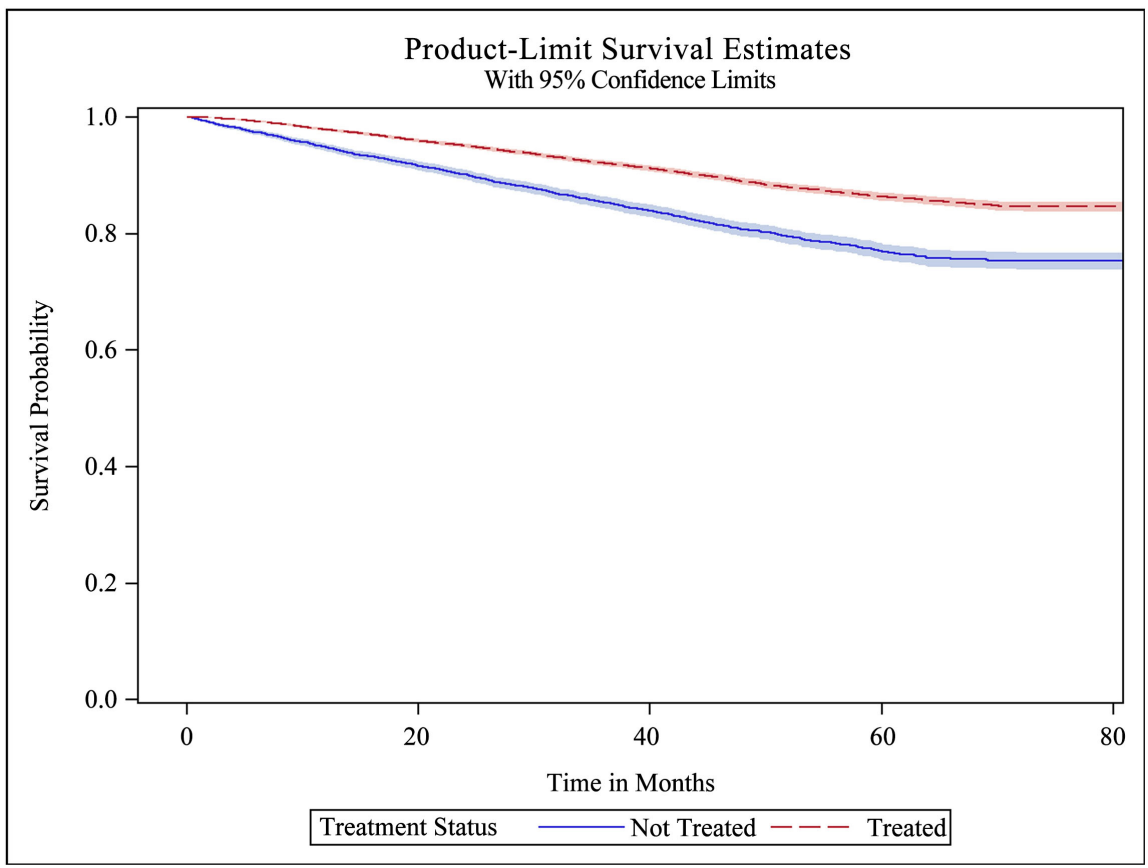

Figure 2. Unadjusted Kaplan-Meier curve of overall survival by treatment status.

Adjusting for demographic and clinical characteristics in Table 3, untreated patients exhibited a $48 \%$ higher risk of death compared to treated patients. Increasing age, male gender, being widowed, poor performance, increasing stage, increasing tumor size, increasing tumor grade, and increasing comorbidity score were significantly associated with higher mortality risks. Receipt of genomic testing was associated with a $45 \%$ reduction in mortality risk. Prior radiotherapy was associated with a $41 \%$ reduction in mortality risk, and black African ancestry was associated with a $16 \%$ reduction in mortality risk compared to whites, after adjusting for all other variables in the model. In a subset of 8967 patients with stage I disease, tumor size $<2.0 \mathrm{~cm}$ and tumor grade 1 or grade 2 ; untreated patients had a $22 \%$ higher risk of death compared to treated patients. In the subset of treated patients, those receiving sequential chemotherapy plus hormonal therapy exhibited a $26 \%(\mathrm{HR}=1.26 ; 95 \% \mathrm{CI}: 1.01-1.58)$ higher risk of death compared to hormonal therapy alone, and there was no mortality risk difference with chemotherapy-only ( $\mathrm{HR}=1.10 ; 95 \% \mathrm{CI}: 0.94-1.30$ ) compared to hormonal therapy, after adjusting for demographic and clinical characteristics (data not shown).

\section{Discussion}

We evaluated the predictors of neoadjuvant/adjuvant therapy receipt and survival among Medicare beneficiaries diagnosed with HR + HER2-breast cancer and found that older patients with more favorable disease characteristics (earlier stage, smaller tumor size, lower tumor grade) were less likely to receive neoadjuvant/adjuvant therapy and had a higher risk of death compared to neoadjuvant/adjuvant treated patients, controlling for other competing causes of mortal- 
ity such as age, co-morbidity burden, and poor performance. Our multivariate survival model also demonstrated that after adjusting for tumor characteristics and neoadjuvant/adjuvant treatment, increasing co-morbidity score was associated with increasing mortality risk, similar to a recent study [22]. The logistic regression model demonstrated that increasing age was associated with lower likelihood of therapy independent of performance status, co-morbidities and tumor characteristics, suggesting that physicians may be under-treating otherwise healthy older patients. Older patients represent a heterogeneous population in terms of fitness, and chronological age alone should not contraindicate life-prolonging or curative treatment.

Table 3. Time-varying Cox model of overall mortality risk in the total population and the stage/size/grade subpopulation.

\begin{tabular}{|c|c|c|c|c|c|c|}
\hline \multirow[b]{2}{*}{ Characteristic } & \multicolumn{3}{|c|}{$\begin{array}{l}\text { All Patients }^{\mathrm{a}} \\
\mathrm{N}=18,470\end{array}$} & \multicolumn{3}{|c|}{$\begin{array}{c}\text { Stage/Size/Grade Subset }{ }^{\mathrm{a}, \mathrm{b}} \\
\qquad \mathrm{N}=8967\end{array}$} \\
\hline & $\mathbf{N}$ & HR & $95 \% \mathrm{CI}$ & $\mathbf{N}$ & HR & $95 \% \mathrm{CI}$ \\
\hline \multicolumn{7}{|l|}{ Subpopulation } \\
\hline Treated (ref) & 13,670 & & & 6175 & & \\
\hline Not Treated & 4800 & 1.476 & $1.35-1.61$ & 2792 & 1.217 & $1.05-1.41$ \\
\hline \multicolumn{7}{|l|}{ Age at Diagnosis } \\
\hline $66-70$ (ref) & 5423 & & & 2747 & & \\
\hline $71-75$ & 4619 & 1.189 & $1.03-1.38$ & 2374 & 1.348 & $1.04-1.75$ \\
\hline $76-80$ & 3871 & 1.615 & $1.40-1.86$ & 1890 & 1.691 & $1.31-2.18$ \\
\hline$>80$ & 4557 & 2.720 & $2.39-3.10$ & 1956 & 3.111 & $2.45-3.95$ \\
\hline \multicolumn{7}{|l|}{ Sex } \\
\hline Female (ref) & 18,300 & & & 8926 & & \\
\hline Male & 170 & 1.507 & $1.11-2.05$ & 41 & 2.928 & $1.55-5.54$ \\
\hline \multicolumn{7}{|l|}{ Race/ethnicity } \\
\hline White (ref) & 15,945 & & & 7911 & & \\
\hline Black & 1269 & 0.843 & $0.73-0.98$ & 450 & 0.872 & $0.63-1.20$ \\
\hline Others & 1256 & 0.776 & $0.65-0.93$ & 606 & 0.713 & $0.51-0.99$ \\
\hline \multicolumn{7}{|l|}{ Marital Status } \\
\hline Married (ref) & 7525 & & & 3973 & & \\
\hline Separated/Divorced & 2001 & 1.067 & $0.91-1.25$ & 978 & 0.943 & $0.71-1.24$ \\
\hline Single & 1648 & 1.129 & $0.97-1.32$ & 718 & 1.027 & $0.76-1.38$ \\
\hline Widowed & 6525 & 1.203 & $1.09-1.33$ & 2928 & 1.244 & $1.05-1.48$ \\
\hline Unknown & 771 & 1.123 & $0.91-1.39$ & 370 & 0.740 & $0.46-1.19$ \\
\hline \multicolumn{7}{|l|}{ Stage at Diagnosis } \\
\hline Stage I (ref) & 11,061 & & & & & \\
\hline Stage II & 5908 & 1.241 & $1.08-1.42$ & & & \\
\hline
\end{tabular}




\section{Continued}

\begin{tabular}{|c|c|c|c|c|c|c|}
\hline Stage III & 1501 & 2.389 & $2.02-2.83$ & & & \\
\hline \multicolumn{7}{|l|}{ Histology } \\
\hline \multicolumn{7}{|l|}{ Grade } \\
\hline Grade 1 (ref) & 5659 & & & & & \\
\hline Grade 2 & 8990 & 1.124 & $1.01-1.25$ & & & \\
\hline Grade $3 / 4$ & 3083 & 1.533 & $1.36-1.73$ & & & \\
\hline Unknown & 738 & 1.282 & $1.05-1.57$ & & & \\
\hline \multicolumn{7}{|l|}{ Tumor Size } \\
\hline$<2.0 \mathrm{~cm}$ & 12,010 & & & & & \\
\hline $2.0-4.9 \mathrm{~cm}$ & 5499 & 1.318 & $1.15-1.51$ & & & \\
\hline$\geq 5.0 \mathrm{~cm}$ & 919 & 1.588 & $1.32-1.91$ & & & \\
\hline Unknown & 42 & 2.772 & $1.67-4.60$ & & & \\
\hline \multicolumn{6}{|c|}{ Poor Performance } & Indicators \\
\hline No (ref) & 16,350 & & & 8217 & & \\
\hline Yes & 2120 & 1.735 & $1.57-1.92$ & 750 & 2.026 & $1.69-2.43$ \\
\hline \multicolumn{7}{|c|}{ NCI Comorbidity } \\
\hline 0 (ref) & 10,481 & & & 5366 & & \\
\hline 1 & 4661 & 1.349 & $1.22-1.49$ & 2260 & 1.708 & $1.43-2.04$ \\
\hline 2 & 1868 & 1.790 & $1.58-2.02$ & 802 & 2.264 & $1.81-2.83$ \\
\hline$\geq 3$ & 1460 & 2.489 & $2.20-2.81$ & 539 & 3.483 & $2.78-4.36$ \\
\hline \multicolumn{7}{|l|}{ RS Assay } \\
\hline No (ref) & 15,460 & & & 7607 & & \\
\hline Yes & 3010 & 0.546 & $0.45-0.66$ & 1360 & 0.745 & $0.55-1.01$ \\
\hline \multicolumn{7}{|l|}{ Radiotherapy } \\
\hline No (ref) & 7785 & & & 3385 & & \\
\hline Yes & 10,685 & 0.591 & $0.54-0.65$ & 5582 & 0.578 & $0.49-0.67$ \\
\hline
\end{tabular}

${ }^{\mathrm{a}}$ Model also includes geographic region, education, income, year of diagnosis, and histology, ${ }^{\mathrm{b}}$ Subset of patients with stage I disease, tumor size $<2.0 \mathrm{~cm}$ and grade $1 / 2$.

The primary element of curative therapy for early-stage breast cancer is surgery. The International Society of Geriatric Oncology (SIOG) panel recommends radiation therapy and adjuvant systemic treatment after breast-conserving surgery in all older patients with breast cancer [23]. The decision to use adjuvant chemotherapy should not be based on age, but on several factors that include comorbidities, functional status, life expectancy, treatment tolerance, patient preference and risk for local recurrence [24] [25]. Although adjuvant systemic therapy has clear benefits in patients with HR+ early breast cancer [26], the treatment rate in our older aged cohort was low. Prior studies show that patients 
over the age of 80 are only half as likely as younger patients to have a discussion about tamoxifen with their physicians due to physician concerns about side effects and treatment adherence [27] [28].

Overall, only $16 \%$ of our study population had genomic testing and this was associated with a higher likelihood of receiving neoadjuvant/adjuvant therapy as well as a $45 \%$ reduction in mortality risk compared to patients who did not have the test. A recent meta-analysis of 15 studies that investigated the impact of RS assays on adjuvant treatment decisions, reported that the additional information provided by the test changed the recommendation for adjuvant treatment in $30 \%$ of cases with the majority being a de-escalation of chemoendocrine therapy to adjuvant endocrine therapy alone [29]. In our study, we found that the rates of sequential therapy with chemotherapy followed by hormonal therapy decreased, while the use of hormonal therapy increased in association with the RS assay. Other trials showed that the benefit of adding chemotherapy to tamoxifen was mainly seen in patients with a high RS and no significant benefit from the addition of chemotherapy was noted in the low and intermediate RS risk groups [11] [30]. However, given the nature of administrative claims data, we were unable to determine results of RS assays and RS score risk groups. In addition, RS assays are usually ordered for patients who are candidates for chemotherapy use. It's possible that the higher mortality risk observed in the non-RS assay group was due to poorer fitness and tolerability concerns that also precluded them from chemotherapy use and RS assay testing. A recent sub analysis from the MINDACT study demonstrated that $24 \%$ of patients with node negative small tumors $(<1 \mathrm{~cm})$ who were identified as clinical low risk, but genomic high risk, derived a benefit from chemotherapy [31]. While another study found that patients with indolent threshold (ultralow-risk) on the 70-gene assay genomic test have a significantly low risk of mortality after surgery without adjuvant systemic treatment [32]. This adds further support that tumor biology is an important factor when considering adjuvant treatments.

Interestingly, our finding that patients of black African ancestry have lower mortality risk compared to white patients is discordant with prior research. African American women have lower incidence of breast cancer but worse survival when compared to white women [33] [34] [35]. Many have postulated that the survival disparity is related to access to healthcare, treatment differences or socioeconomic factors [36] [37] [38] and race alone is seen as an independent predictor of survival [39]. However, a more recent SEER-Medicare analysis confirmed that after matching patients on treatment as well as demographics, comorbidities, and tumor characteristics at presentation, there was very little difference in survival between black and white women [40].

Receipt of treatment and survival varied by marital status, similar to patterns observed in prior oncology research [41] [42]. In the current study unmarried status, especially widowhood were predictive of not receiving neoadjuvant/adjuvant treatment and was associated with a higher risk of mortality. Male 
patients made up about $1 \%$ of our study population [2] and were more likely to receive neoadjuvant/adjuvant therapy, but had a higher mortality risk compared to females. Breast cancer in males was long thought to have a less favorable outcome than in females [43] [44]. However, others have found little gender difference in survival when matched for age, stage and grade [3] [45] [46]. Further research is warranted to better understand the disparity of nonclinical factors on receipt of cancer therapy to ensure appropriate cancer care and improve outcomes for all patients.

\section{Strengths \& Limitations}

A major strength of this study is that it is a large population-based sample that allows longitudinal evaluation of outcomes that may not be possible in clinical trials. Further, cancer occurs disproportionately in older patients and the dataset is a valuable tool to study patients who have been historically underrepresented in clinical trials. However, it is important to acknowledge the limitations of this retrospective, observational study using administrative claims data. The first limitation relates to missing data for HER2 status prior to the year 2010. Medicare does not pay for targeted therapy without a positive HER2 test result so we used the presence of claims for HER2 targeted therapy as a proxy for HER2+ status and the absence of these claims to indicate HER2-status. The magnitude and direction of the potential bias introduced by the missing data are unknown as patients who tested HER2+ and did not receive targeted therapy could have been misclassified into the HER2-group. It is possible that because untreated HER2+ cancers pose a higher morality risk, this potential misclassification would bias the results by increasing apparent mortality in the untreated group.

The second limitation involves an imputation method for performance status since the database does not include a direct measure for ECOG performance status. We identified diagnostic and procedure codes for specific healthcare resource use that have been shown to be predictive of functional status. Although this surrogate for poor performance may not adequately assess functional status for all patients in our study, it improves the validity of this observational study by attempting to control for such an important confounding variable.

Third, Medicare claims data are created for payment purposes and although we are able to detect who received the RS assay, we do not know the results of the test or patients RS risk groups. The decision to forgo therapy in the older individual often involves many factors such as patient and family preferences, and/or physician concerns for poor tolerance of therapy due to comorbidity and declining organ function [47]; and the SEER-Medicare database does not take into account these physician and patient preferences with regard to treatment. Similarly, patients in the oral hormonal therapy group may be susceptible to misclassification bias as the data derived from administrative claims provide information on medications that were filled, but does not reflect actual medication exposure. To minimize the impact of these potentially misclassified individuals 
in the oral hormonal therapy group, we required evidence of at least two prescriptions and no gaps of at least 120 days between prescriptions to increase the likelihood that patients are actually taking the medicine.

Fourth, the use of overall survival as an endpoint should be interpreted with caution. Although overall survival is the most reliable and available survival measure, it may not be specific enough to provide information on survival related to breast cancer treatment. Therefore, it's not clear if the higher survival we observed among treated patients is due to fewer deaths from other competing causes or fewer deaths from breast cancer treatment. Finally, the data presented here are limited to Medicare enrollees and patients enrolled in HMO represent a gap in the SEER-Medicare database. HMO enrollees tend to be younger and healthier than beneficiaries in fee-for-service plans resulting in a biased loss of information using the Medicare claims data [16].

\title{
6. Conclusion
}

Older patients with HR + HER2-resected breast cancer who have favorable disease characteristics (earlier stage, smaller tumor size and lower tumor grade) were less likely to receive adjuvant/neoadjuvant therapy with hormonal and/or chemotherapy. Untreated patients with favorable disease characteristics had a significantly higher risk of death compared to treated patients, after controlling for comorbidities, poor performance and other patient characteristics. Additional research is needed to determine why adjuvant/neoadjuvant therapies are omitted among the older "fit" breast cancer patient population.

\section{Author Contributions}

\author{
Study Concepts: SS-H, CR; \\ Study Design: SS-H, AS, PC, FM, CR; \\ Data Acquisition: SS-H, CR; \\ Quality Control of Data and Algorithms: SS-H, FM; \\ Data Analysis and Interpretation: SS-H, AS, PC, FM, CR; \\ Statistical Analysis: SS-H, FM; \\ Manuscript Preparation: SS-H; \\ Manuscript Editing: SS-H, AS, PC, FM, CR; \\ Manuscript Review: SS-H, AS, PC, FM, CR.
}

\section{Acknowledgements}

The authors would like to thank Mr. Hans-Peter Goertz for thoughtful review of the manuscript. This study was supported by Genentech, Inc. of South San Francisco, USA, a member of the Roche Group. This study used the linked SEER-Medicare database. We acknowledge the efforts of the Applied Research Program, NCI (Bethesda, MD), the Office of Information Services and the Office of Strategic Planning, Health Care Financing Administration (Baltimore, MD), Information Management Services, Inc. (Silver Spring, MD), and the Surveil- 
lance, Epidemiology, and End Results (SEER) Program tumor registries in the creation of the SEER-Medicare database. The interpretation and reporting of these data are the sole responsibility of the authors.

\section{Conflicts of Interest}

AS, PC and CR are employees of Genentech and shareholders of Roche. SSH and FM work for Q.D. Research in a research and consulting capacity.

\section{References}

[1] Jemal, A., et al. (2010) Cancer Statistics, 2010. CA: A Cancer Journal of Clinicians, 60, 277-300. https://doi.org/10.3322/caac.20073

[2] Siegel, R.L., Miller, K.D. and Jemal, A. (2017) Cancer Statistics, 2017. CA: A Cancer Journal of Clinicians, 67, 7-30. https://doi.org/10.3322/caac.21387

[3] Anderson, W.F., et al. (2004) Is Male Breast Cancer Similar or Different than Female Breast Cancer? Breast Cancer Research and Treatment, 83, 77-86. https://doi.org/10.1023/B:BREA.0000010701.08825.2d

[4] Howlader, N., et al. (2014) US Incidence of Breast Cancer Subtypes Defined by Joint Hormone Receptor and HER2 Status. JNCI: Journal of the National Cancer Institute, 106. https://doi.org/10.1093/jnci/dju055

[5] Crivellari, D., et al. (2000) Burdens and Benefits of Adjuvant Cyclophosphamide, Methotrexate, and Fluorouracil and Tamoxifen for Elderly Patients with Breast Cancer: The International Breast Cancer Study Group Trial VII. Journal of Clinical Oncology, 18, 1412-1422. https://doi.org/10.1200/JCO.2000.18.7.1412

[6] Jemal, A., et al. (2017) Annual Report to the Nation on the Status of Cancer, 1975-2014, Featuring Survival. Journal of the National Cancer Institute, 109. https://doi.org/10.1093/jnci/djx030

[7] Narod, S., Iqbal, J. and Miller, A.B. (2015) Why Have Breast Cancer Mortality Rates Declined? Journal of Cancer Policy, 5, 8-17.

https://doi.org/10.1016/j.jcpo.2015.03.002

[8] National Comprehensive Cancer Network, NCCN Clinical Practice Guidelines in Oncology: Breast Cancer, Version 2, National Comprehensive Cancer Network, Editor. 2013: Fort Washington, PA.

[9] Henry, N.L., et al. (2016) Role of Patient and Disease Factors in Adjuvant Systemic Therapy Decision Making for Early-Stage, Operable Breast Cancer: American Society of Clinical Oncology Endorsement of Cancer Care Ontario Guideline Recommendations. Journal of Clinical Oncology, 34, 2303-2311.

https://doi.org/10.1200/JCO.2015.65.8609

[10] Paik, S., et al. (2004) A Multigene Assay to Predict Recurrence of Tamoxifen-Treated, Node-Negative Breast Cancer. New England Journal of Medicine, 351, 2817-2826. https://doi.org/10.1056/NEJMoa041588

[11] Paik, S., et al. (2006) Gene Expression and Benefit of Chemotherapy in Women with Node-Negative, Estrogen Receptor-Positive Breast Cancer. Journal of Clinical Oncology, 24, 3726-3734. https://doi.org/10.1200/JCO.2005.04.7985

[12] Hutchins, L.F., et al. (1999) Underrepresentation of Patients 65 Years of Age or Older in Cancer-Treatment Trials. New England Journal of Medicine, 341, 2061-2067. https://doi.org/10.1056/NEJM199912303412706

[13] Du, X. and Goodwin, J.S. (2001) Patterns of Use of Chemotherapy for Breast Cancer 
in Older Women: Findings from Medicare Claims Data. Journal of Clinical Oncology, 19, 1455-1461. https://doi.org/10.1200/JCO.2001.19.5.1455

[14] Extermann, M., Balducci, L. and Lyman, G.H. (2000) What Threshold for Adjuvant Therapy in Older Breast Cancer Patients? Journal of Clinical Oncology, 18, 1709-1717. https://doi.org/10.1200/JCO.2000.18.8.1709

[15] Newschaffer, C.J., et al. (1996) The Effect of Age and Comorbidity in the Treatment of Elderly Women with Nonmetastatic Breast Cancer. Arch Intern Med, 156, 85-90. https://doi.org/10.1001/archinte.1996.00440010103014

[16] Warren, J.L., et al. (2002) Overview of the SEER-Medicare Data: Content, Research Applications, and Generalizability to the United States Elderly Population. Medical Care, 40, IV-3-18. https://doi.org/10.1097/00005650-200208001-00002

[17] Davidoff, A.J., et al. (2010) Chemotherapy and Survival Benefit in Elderly Patients with Advanced Non-Small-Cell Lung Cancer. Journal of Clinical Oncology, 28, 2191-2197. https://doi.org/10.1200/JCO.2009.25.4052

[18] Klabunde, C.N., et al. (2007) A Refined Comorbidity Measurement Algorithm for Claims-Based Studies of Breast, Prostate, Colorectal, and Lung Cancer Patients. Annals of Epidemiology, 17, 584-590. https://doi.org/10.1016/j.annepidem.2007.03.011

[19] Charlson, M.E., et al. (1987) A New Method of Classifying Prognostic Comorbidity in Longitudinal Studies: Development and Validation. Journal of Chronic Diseases, 40, 373-383. https://doi.org/10.1016/0021-9681(87)90171-8

[20] Dinan, M.A., et al. (2015) Association between Use of the 21-Gene Recurrence Score Assay and Receipt of Chemotherapy among Medicare Beneficiaries with Early-Stage Breast Cancer, 2005-2009. JAMA Oncology, 1, 1098-1109. https://doi.org/10.1001/jamaoncol.2015.2722

[21] Suissa, S. (2008) Immortal Time Bias in Pharmaco-Epidemiology. American Journal of Epidemiology, 167, 492-499. https://doi.org/10.1093/aje/kwm324

[22] Kimmick, G.G., et al. (2018) Risk of Cancer Death by Comorbidity Severity and Use of Adjuvant Chemotherapy among Women with Locoregional Breast Cancer. Journal of Geriatric Oncology, 9, 214-220. https://doi.org/10.1016/j.jgo.2017.11.004

[23] Biganzoli, L., et al. (2012) Management of Elderly Patients with Breast Cancer: Updated Recommendations of the International Society of Geriatric Oncology (SIOG) and European Society of Breast Cancer Specialists (EUSOMA). The Lancet Oncology, 13, e148-e160. https://doi.org/10.1016/S1470-2045(11)70383-7

[24] Cutuli, B. (2009) Breast Cancer Irradiation in Elderly. Cancer Radiothérapie, 13, 615-622. https://doi.org/10.1016/j.canrad.2009.06.027

[25] Tesarova, P. (2012) Breast Cancer in the Elderly-Should It Be Treated Differently? Reports of Practical Oncology and Radiotherapy, 18, 26-33. https://doi.org/10.1016/j.rpor.2012.05.005

[26] Early Breast Cancer Trialists' Collaborative, G. (2005) Effects of Chemotherapy and Hormonal Therapy for Early Breast Cancer on Recurrence and 15-Year Survival: An Overview of the Randomised Trials. The Lancet, 365, 1687-1717. https://doi.org/10.1016/S0140-6736(05)66544-0

[27] Blackman, S.B., et al. (2002) Advanced Age and Adjuvant Tamoxifen Prescription in Early-Stage Breast Carcinoma Patients. Cancer, 95, 2465-2472. https://doi.org/10.1002/cncr.10985

[28] Silliman, R.A., et al. (2002) Adjuvant Tamoxifen Prescription in Women 65 Years and Older with Primary Breast Cancer. Journal of Clinical Oncology, 20, 2680-2688. 
https://doi.org/10.1200/JCO.2002.08.137

[29] Augustovski, F., et al. (2015) Decision-Making Impact on Adjuvant Chemotherapy Allocation in Early Node-Negative Breast Cancer with a 21-Gene Assay: Systematic Review and Meta-Analysis. Breast Cancer Research and Treatment, 152, 611-625. https://doi.org/10.1007/s10549-015-3483-3

[30] Albain, K.S., et al. (2010) Prognostic and Predictive Value of the 21-Gene Recurrence Score Assay in Postmenopausal Women with Node-Positive, Oestrogen-Receptor-Positive Breast Cancer on Chemotherapy: A Retrospective Analysis of a Randomised Trial. The Lancet Oncology, 11, 55-65.

https://doi.org/10.1016/S1470-2045(09)70314-6

[31] Tryfonidis, K., et al. (2017) Not All Small Node Negative (pT1abN0) Breast Cancers Are Similar: Outcome Results from an EORTC 10041/BIG 3-04 (MINDACT) Trial Substudy. Annals of Oncology, 28, v605-v649. https://doi.org/10.1093/annonc/mdx440.003

[32] Esserman, L.J., et al. (2017) Use of Molecular Tools to Identify Patients with Indolent Breast Cancers with Ultralow Risk over 2 Decades. JAMA Oncology, 3, 1503-1510. https://doi.org/10.1001/jamaoncol.2017.1261

[33] Chu, K.C., Lamar, C.A. and Freeman, H.P. (2003) Racial Disparities in Breast Carcinoma Survival Rates: Seperating Factors That Affect Diagnosis from Factors That Affect Treatment. Cancer, 97, 2853-2860. https://doi.org/10.1002/cncr.11411

[34] Polite, B.N. and Olopade, O.I. (2005) Breast Cancer and Race: A Rising Tide Does Not Lift All Boats Equally. Perspectives in Biology and Medicine, 48, S166-S175. https://doi.org/10.1353/pbm.2005.0027

[35] Chlebowski, R.T., et al. (2005) Ethnicity and Breast Cancer: Factors Influencing Differences in Incidence and Outcome. Journal of the National Cancer Institute, 97, 439-448. https://doi.org/10.1093/jnci/dji064

[36] Breen, N., et al. (1999) The Relationship of Socio-Economic Status and Access to Minimum Expected Therapy among Female Breast Cancer Patients in the National Cancer Institute Black-White Cancer Survival Study. Ethnicity \& Disease, 9, 111-125.

[37] Bradley, C.J., Given, C.W. and Roberts, C. (2002) Race, Socioeconomic Status, and Breast Cancer Treatment and Survival. Journal of the National Cancer Institute, 94, 490-496. https://doi.org/10.1093/jnci/94.7.490

[38] Gordon, N.H. (2003) Socioeconomic Factors and Breast Cancer in Black and White Americans. Cancer and Metastasis Reviews, 22, 55-65. https://doi.org/10.1023/A:1022212018158

[39] Newman, L.A., et al. (2006) Meta-Analysis of Survival in African American and White American Patients with Breast Cancer: Ethnicity Compared with Socioeconomic Status. Journal of Clinical Oncology, 24, 1342-1349. https://doi.org/10.1200/JCO.2005.03.3472

[40] Silber, J.H., et al. (2013) Characteristics Associated with Differences in Survival among Black and White Women with Breast Cancer. JAMA, 310, 389-397. https://doi.org/10.1001/jama.2013.8272

[41] Kravdal, O. (2001) The Impact of Marital Status on Cancer Survival. Social Science \& Medicine, 52, 357-368. https://doi.org/10.1016/S0277-9536(00)00139-8

[42] Lai, H., et al. (1999) The Effect of Marital Status on Survival in Late-Stage Cancer Patients: An Analysis Based on Surveillance, Epidemiology, and End Results (SEER) Data, in the United States. International Journal of Behavioral Medicine, 6, 150-176. https://doi.org/10.1207/s15327558ijbm0602_4 
[43] Adami, H.O., et al. (1985) Long-Term Survival in 406 Males with Breast Cancer. British Journal of Cancer, 52, 99-103. https://doi.org/10.1038/bjc.1985.155

[44] Salvadori, B., et al. (1994) Prognosis of Breast Cancer in Males: An Analysis of 170 Cases. European Journal of Cancer, 30A, 930-935. https://doi.org/10.1016/0959-8049(94)90117-1

[45] Borgen, P.I., et al. (1997) Carcinoma of the Male Breast: Analysis of Prognosis Compared with Matched Female Patients. Annals of Surgical Oncology, 4, 385-388. https://doi.org/10.1007/BF02305550

[46] Scott-Conner, C.E., et al. (1999) An Analysis of Male and Female Breast Cancer Treatment and Survival among Demographically Identical Pairs of Patients. Surgery, 126, 775-780. https://doi.org/10.1016/S0039-6060(99)70135-2

[47] Dale, D.C. (2003) Poor Prognosis in Elderly Patients with Cancer: The Role of Bias and Undertreatment. The Journal of Supportive Oncology, 1, 11-17. 